\title{
Layered perspectives: Students' experiences with three levels of partnership
}

\author{
Hannah Jardine, Center for Teaching, Research \& Learning, America University, Washington, \\ DC, USA.
}

Danielle Pafe, Teaching and Learning Transformation Center, University of Maryland-College Park, USA.

Julianna Burns, University of Maryland-College Park, USA.

Jenna Feeley, University of Maryland-College Park, USA.

Contact: jardine.hannah.e@gmail.com

Community and connection are critical for a successful undergraduate experience; connection to fellow students, mentors, faculty, and staff- and student-faculty partnership programs can provide meaningful community. The Academic Peer Mentoring Program (AMP) at University of Maryland aims to create community by integrating undergraduate students into courses and research settings to work with faculty as part of an instructional team. Students in courses supported by peer educators build connections with other students in the course, the peer educators, and the faculty. Peer educators experience a sense of community with each other, their fellow students, and the faculty members they partner with. On another level, the program creates a community for peer educators across different courses. This essay presents the unique perspectives of two students, Julianna and Jenna, who experienced the AMP community through three different levels of partnership over three years.

Julianna and Jenna were first introduced to AMP in Spring 2018 as students in a peer educator-supported course in the First-Year Innovation and Research Experience program (FIRE). FIRE partners with AMP to integrate peer educators into their research-based courses. In FIRE, students engage in authentic research experiences guided by faculty and peer educators. After students complete the three-semester research experience, they may apply to support incoming students as peer educators, which is what Julianna and Jenna did in Spring 2019. As peer educators, they worked closely with faculty on research and advanced their leadership skills by training and mentoring new students.

During their first semester in the role, Julianna and Jenna enrolled in the one-credit course, Fundamentals of Peer Education, that all new peer educators take. In that course, they learned about principles of effective teaching, learning, and mentorship and developed professional skills to support fellow students. They continued as peer educators in Fall 2019. In Spring 2020, they applied to serve as instructional partners alongside two staff members 
Hannah and Danielle, who, at the time, were the AMP coordinators and co-teachers of the Fundamentals course.

As the coordinators for AMP, Hannah and Danielle worked to integrate notions of student-faculty partnership into the program by moving from a focus on student training to a focus on student-instructor connections (Jardine, 2020). It seemed hypocritical and a missed opportunity not to partner with students as they made changes to the program. In Spring 2020, Hannah and Danielle recruited three former peer educators to partner with them for the first time, including Julianna and Jenna, with the expectation that these experienced peer educators would be able to provide valuable support to new peer educators as well as help the program leaders better understand the peer educator experience. Thus, Julianna and Jenna participated in AMP for three years-as students in a course supported by peer educators, as peer educators, and as part of the program-level instructional team.

In this essay, our instructional team reflects on the experience of working together over the Spring 2020 semester, and Julianna and Jenna share their experience of partnership in these three different ways. They first consider how they benefited from being in a course supported by student-faculty instructional teams. Then, they analyze their experience as part of the student-faculty instructional team, including the benefits and challenges of the studentfaculty partnership dynamic. Lastly, they share what it was like to work with new peer educators and partner with the program coordinators. Hannah and Danielle, the AMP coordinators, consider all they learned from working alongside these insightful students. The entire team also analyzes how working together during the Spring 2020 semester was particularly crucial for maintaining the community and connection that AMP aims for as they faced a sudden transition to remote instruction during a global pandemic.

\section{STUDENTS REFLECT ON THREE LEVELS OF PARTNERSHIP}

The following sections are written from the first-person perspective of Julianna and Jenna.

\section{First perspective: As students in a course supported by student-faculty instructional teams}

Transitioning from small high schools to a large university was intimidating for us; we imagined that large class sizes would make it difficult to create meaningful relationships with faculty. Thankfully, the FIRE program maintained small class sizes supported by peer educators, which allowed us to create meaningful relationships with faculty and other students. The peer educators provided an experienced student perspective, helped us review faculty lectures and instructions, and observed our first experiments in order to provide feedback. This support improved our ability to fully grasp and appreciate the scientific research we were performing.

As students in a course supported by student-faculty instructional teams, we experienced a unique opportunity by having greater access to the faculty member and the peer educators. Since peer educators were able to answer our general questions, faculty were more available to have targeted, in-depth conversations about research. We felt that the peer educators set an important example of what a positive faculty-student relationship might look like; we observed them continuing to learn from faculty about research theory, basic lab skills, and career opportunities. Beyond the research and course material, we were able to ask both 
faculty and peer educators detailed questions about everything from course selection to career aspirations. We believe that being a part of a course and program supported by student-faculty instructional teams during our first year at a university was invaluable to our success and kept us motivated and inspired to continue in research and support our peers as we moved forward.

\section{Second perspective: As part of the student-faculty instructional team}

Because we appreciated and looked up to the peer mentors who worked with us when we were in our first-year courses, we decided to pursue becoming peer mentors ourselves. As peer mentors, we were expected to answer student questions, observe and guide students as they worked through lab protocols, and work alongside our faculty partners to aid students' learning. In addition, we conducted research ourselves and maintained lab upkeep such as inventory, ordering, and cleaning, which are all roles traditionally held by a faculty member or graduate student. Because we were entrusted with these responsibilities, we felt we gained an authentic and realistic research experience, which confirmed our own interest in pursuing research as a career.

Knowing that faculty trusted us to explain the lab theory and protocols to students encouraged us to delve into the material more deeply. We evaluated where we could improve or alter experimental protocols, and we shared this insight with our faculty partners. In addition, we learned how to interact with students in a way that motivated them to grow as student researchers. For example, if a student made a mistake, we would ask them detailed questions to help them think through each step and troubleshoot the error. We did this in a non-dominating or non-intimidating way so that the student did not feel embarrassed about making a mistake. If a student seemed upset or disappointed about their mistake, we found that it was useful to share our own trials and errors; this transparency reiterated the message that making mistakes is a natural part of the learning process.

As peer mentors, we maintained trust and open communication with our faculty partners. In our weekly meetings, we discussed accomplishments and challenges, including issues that occurred in the lab, which allowed our instructional team to make adjustments to better help students. We used a variety of formal and informal communication tools, such as emails and group messages, to communicate pertinent information. By maintaining open, informal communication between peer educators and faculty partners, we felt that we could raise any question, concern, or mistake that arose and could express and discuss our ideas without judgement.

However, one challenge that came out of the informal environment was an overly informal attitude from some peer educators. The program emphasis on collaboration encouraged peer mentors to support one another as we established stronger communication and professional behaviors. We recommend that students and faculty spend time at the start of their partnership discussing expectations and boundaries to build a strong foundation for the new partnership. Overall, we feel that open communication and feedback led to a cohesive and meaningful student-faculty partnership where we could engage deeper with the research, better support students in the course, and develop professional and leadership skills.

Because they granted us responsibility in the lab and treated us like colleagues, it was clear to us that our faculty partners believed in our ability to guide students through their first year of academic research. This feeling of respect and appreciation fueled us to take the 
responsibility of teaching students seriously; instead of simply helping students follow instructions, we helped them appreciate academic research.

Third perspective: Supporting new students working on student-faculty instructional teams

When we were asked to support AMP as student partners, we were very excited by the opportunity to engage with the program at an even higher level by supporting new peer educators. Similar to when we were peer educators, we felt we were valued members of the instructional team because of the open communication and collaborative dynamics that Hannah and Danielle established. Our insights and suggestions regarding the content and structure of the Fundamentals course were taken into consideration before, during, and after the course, which led us to feel increased motivation and an increased sense of responsibility.

Being part of the instructional team for the Fundamentals course allowed us to apply everything we had learned as students in courses supported by peer educators and as peer educators. Hannah and Danielle gave us the opportunity to develop resource pages on a topic of our choosing that we thought would be valuable for peer educators in their first semester. Having autonomy and full control over development of resources instilled in us a sense of pride and confidence. We were also responsible for reviewing students' written reflections, which helped us learn how to give constructive feedback. Having experienced the peer educator role the previous year, we were able to help incoming peer educators navigate unfamiliar and often challenging situations. Our feedback was well received by the peer educators, and we built connections with them by sharing our experiences.

One task we initially struggled with was grading, specifically how to balance being empathetic to the students while upholding high expectations. In retrospect, it might have been beneficial if the entire instructional team had graded the first few assignments together. This would have allowed for developing a uniform and concrete method of grading from the start.

One course assignment that we implemented was a progress meeting reflection, which required peer educators to meet with their faculty partner to get feedback on their performance and then write a reflection with plans for next steps. In FIRE, we had routine check-ins with our faculty partners during which we received constructive criticism and could ask questions and discuss challenges privately. These meetings helped us to build deeper relationships with our faculty partners and grow professionally. Therefore, when Hannah and Danielle voiced the idea of making this a requirement for every new peer educator taking the Fundamentals course, we thought it would be a great opportunity for the peer educators to develop their leadership skills. When reading students' reflections, we recognized how much the peer educators benefited from this formal check-in with their faculty partner, and we felt honored knowing that we had influence over their positive experiences.

In the very difficult time of the unfolding of the COVID-19 pandemic midway through the semester, we appreciated the opportunity to observe Hannah's and Danielle's commitment to students and resiliency as instructors. Although certain aspects of the course had to be altered, we remained dedicated to providing a space for peer educators to discuss the trials they were facing in such an unusual time. Being part of the instructional team during the pandemic allowed us to practice dynamic (as opposed to static) education. This challenge presented another opportunity to teach students resiliency, patience, and the importance of 
self-care by helping them persist through setbacks and find inspiration by hearing about the successes of others, and we learned these important lessons as well. While this role often felt overwhelming as we juggled many new responsibilities, it was worth all the time and effort to make a meaningful contribution to the course.

We both feel that we expanded our leadership skills and gained invaluable professional development throughout the experience of serving as student partners for AMP. In terms of future changes, we recommended to Hannah and Danielle that they hold formal progress meetings with students like us who are part of the instructional team. Although we checked in regularly during our weekly meetings, we believe formal meetings and feedback on our performance would have allowed for even further growth as leaders throughout the semester.

\section{PROGRAM COORDINATORS REFLECT ON WORKING WITH STUDENT PARTNERS}

This section is written from the first-person perspective of Hannah and Danielle.

Our experience working with Julianna and Jenna has been more insightful, valuable, and enjoyable than we could have imagined. Throughout the semester, our student partners were able to share valuable insight with us, as the peer educators were more comfortable being vulnerable and transparent when discussing their progress with fellow students. In addition, Julianna and Jenna taught us about the unique role of the peer educators in FIRE so that we could cater our materials better to peer educators in research settings. We felt humbled and inspired by their commitment to supporting other students, willingness to develop and adapt resources for the program, and dedication to learning about the faculty perspective.

By working with Julianna and Jenna, we were able to make meaningful adjustments to the Fundamentals course and better attend to current peer educators' personal and professional needs. For example, while developing the new progress meeting reflection assignment, Julianna and Jenna lent their ideas about how students may experience this meeting and assignment. This conversation led to a scaffolded reflection assignment that focused on a growth-mindset approach to receiving constructive feedback, which we felt was much more valuable than what we might have developed on our own. Collaborating with Julianna and Jenna also made us feel more engaged, as it was energizing to talk through ideas and create materials together. Working with them led us to continuously question and consider the student experience and how accessible and relatable the course content was.

Julianna and Jenna also brought their unique perspectives when creating new supplementary resources for peer educators on time management and professionalism. When we gave them the responsibility of generating resources for students, we felt like they were experimenting with the role of instructor in a different way than many of their other duties. Although at first it felt slightly uncomfortable to give up control over course materials and resources, it was exciting to see the result that came from giving them complete ownership over one aspect of the course.

Particular to the Spring 2020 semester, the transition to online learning in the midst of a pandemic was a challenge we needed to adapt to quickly, and our student partners were invaluable in our decision making. At first, it felt more complicated to include our student partners in this process, as the amount of time needed to seek their perspectives and process those perspectives together felt a bit burdensome in the midst of so many other changes, and we also wanted to be respectful of their time. However, we were not as in tune with the 
emotions and workload of students as our student partners. Hearing what they would like to see in their own courses during the rapid shift online was critical to our shared decision making. Together, we thought through how to shift assignments and what to say in messaging to students about our expectations regarding balancing personal and work commitments with deadlines and quality of submissions.

We intend to continue to have new student partners work with AMP and the Fundamentals course every semester. One area we want to improve is our process for assessing and giving feedback to our student partners. In Spring 2020, we checked in with our student partners informally and shared expectations in ongoing weekly team meetings. In future semesters, although it would require additional time commitments, individual meetings with each partner at a few designated times during the semester would provide more individualized coaching and would allow us to learn more about their specific interests. It is exciting to think of the projects that could arise from a more individualized approach to partnership where we build on the strengths and perspectives of each student.

Additionally (for Hannah as the program coordinator), going through the process of partnership, while at the same time promoting partnership with the instructors involved with $A M P$, helped to improve the support provided to other instructors. Experiencing firsthand the process of recruiting student partners, negotiating responsibilities, scheduling, meeting, building relationships, and developing trust helped to improve the resources created for instructors working with peer educators. Going through the process helped turn hypothetical suggestions into suggestions based on experience.

\section{SUMMARY AND LOOKING FORWARD}

We-Hannah, Danielle, Julianna, and Jenna-have all thoroughly enjoyed working together and benefited greatly from our partnership. Through writing this essay collaboratively and considering the different perspectives and versions of partnership that students, faculty, and staff might experience, we have learned a lot about ourselves as teachers and learners. We recognize the value of applying personal experience to support others and that much more can be accomplished when we trust others to take on roles outside of the norm. We all plan to apply the lessons from these experiences to new leadership and mentorship roles. The suggestions and ideas that our instructional team discussed will undoubtedly lead to improvements in AMP and the Fundamentals course for the next semester and future semesters. We are grateful for this opportunity to form these different connections, give back to our institution through establishing a more welcoming and tight-knit learning community, and share our experiences with the broader higher educational community so that others may be inspired to pursue and enhance their partnerships.

\section{NOTE ON CONTRIBUTORS}

Hannah Jardine currently supports educational development at the Center for Teaching, Research, and Learning at American University. She previously worked for the University of Maryland Teaching and Learning Transformation Center, where she coordinated the Academic Peer Mentor program. Her research focuses on the process and outcomes of positioning students as instructional partners, particularly in undergraduate STEM. 
Danielle Pafe is a Coordinator of Collaborative Learning at the University of Maryland Teaching and Learning Transformation Center. She specializes in academic support programs and currently works with undergraduate peer leaders to improve peer-to-peer interactions in historically difficult courses.

Julianna Burns graduated from the University of Maryland, College Park. She majored in Biology with a concentration in Ecology and Evolution, and she minored in Soil Science. She plans to pursue a Masters in Soil Sciences and purse a career with a multidisciplinary focus in both Biology and Agriculture.

Jenna Feeley graduated from the University of Maryland, College Park with a Bachelor of Science in Nutritional Science. Jenna is now a Post-Baccalaureate Fellow at NIH and is pursuing a career in medicine and research.

\section{REFERENCES}

Jardine, H. E. (2020). Integrating partnership into an academic peer mentoring program: Moving from a focus on student training to a focus on building student-instructor connections. Teaching and Learning Together in Higher Education, 1(29).

https://repository.brynmawr.edu/tlthe/vol1/iss29/2 